\title{
EVOLUTION OF CONTACT SYSTEMS
}

\author{
O. VILHU and T. RAHUNEN \\ Observatory and Astrophysics Laboratory, University of Helsinki, Finland
}

\begin{abstract}
The evolution of contact binaries with common convective envelopes has been studied. Gravitational energy due to the mass exchange is taken into account in both stars, which turned out to be of crucial importance. Three qualitatively different evolutionary tracks have been found for an initial system $1.2 M_{\odot}+1.0 M_{\odot}$. One of these corresponds to those found by Moss (1971), in which mass transfers, on a long time scale $\left(\approx 5 \times 10^{8} \mathrm{yr}\right)$, from the less massive component to the heavier. The second is similar to the solution found by Hazlehurst and Meyer-Hofmeister (1973), in which the evolution proceeds towards equal masses in a much shorter time scale $\left(\approx 2 \times 10^{7} \mathrm{yr}\right)$. The third possibility is similar to the first one, but its time scale is 10 times shorter $\left(\approx 5 \times 10^{7} \mathrm{yr}\right)$. The true evolutionary track may well oscillate randomly around the different solutions (rapid period changes).
\end{abstract}

The evolution of contact low mass systems has been investigated using equal entropies (by the luminosity transfer method) in the convective envelopes of the components. Hence the models are based on the old Lucy model and on the luminosity-transfer picture developed mainly by the English group (Hazlehurst, Whelan, Moss and others). Further, the surface of the common envelope was allowed to move freely between the inner and outer critical surfaces. The evolutionary programmes, kindly given by Dr Paczynski for this purpose, were modified and used in the computations.

The initial equilibrium models consisted of a homogeneous secondary and an evolved primary, because homogeneous systems with equal entropies would be impossible for the normal chemical composition used. The starting systems can be thought to result from a Case A evolution. This type of evolution sooner or later leads to a contact system. Further, in this case the ages of our systems depend on the initial masses of the Case A primaries. If the initial mass-ratios are sufficiently large, the actual ages of our models may be quite small.

Similar evolutionary calculations have been performed earlier by Moss (1971) and by Hazlehurst and Meyer-Hofmeister (1973, H\&M-H). Moss finds that, in the course of evolution, mass transfers on a nuclear time scale from the less massive component to the heavier one. In addition, the evolutionary tracks derived by Moss in the colour-period diagram nicely cover the observed region of W UMa-systems. On the contrary, H\&M-H find that the evolution proceeds much more rapidly and towards equal masses. The most significant difference in the above mentioned works was that Moss did not take into account for the secondary the gravitational energy due to the mass transfer. However, for the slow mass-transfer rates found by Moss, the gravitational energy terms would be unimportant.

The present work investigates the reason for the discrepancy between the results of Moss and of H\&M-H. First several thermal equilibrium systems were constructed where the contact conditions (equal entropies $S$ and the same equipotential $C$ in both components) were fulfilled, at least approximately. These initial systems were then evolved with different rates for the mass transfer $\dot{M}$ and for the luminosity transfer change $|\Delta \dot{L}|$, in a similar way to H\&M-H. From these grids of models we tried to find values of $\dot{M}$ and $|\Delta \dot{L}|$ with the same changes of $S$ and $C$ for both components. This would guarantee that the contact conditions do not break during the evolution. The changes of $S$ and $C$ were taken from the interval $0.02 M_{\odot}-0.04 M_{\odot}$ (mass transfer from the beginning). 
The situation is illustrated in Figure 1. The solution for the rate $\dot{M}$ should lie on the crossing point of the curves and the abscissae. It can be seen that in all cases small negative $\dot{M}$ (mass transfer from the less massive component to the heavier) is the solution. This corresponds to Moss's solutions. In addition, the curves indicate that two other solutions probably also exist: more rapid mass transfer from the primary to the secondary, and vice versa. The large positive $\dot{M}$ would correspond to the solution given by $\mathbf{H} \& \mathbf{M}-\mathbf{H}$.

From these preliminary computations it appeared that the solutions are fairly sensitive to the proper treatment of the convective core (which in many computational programmes
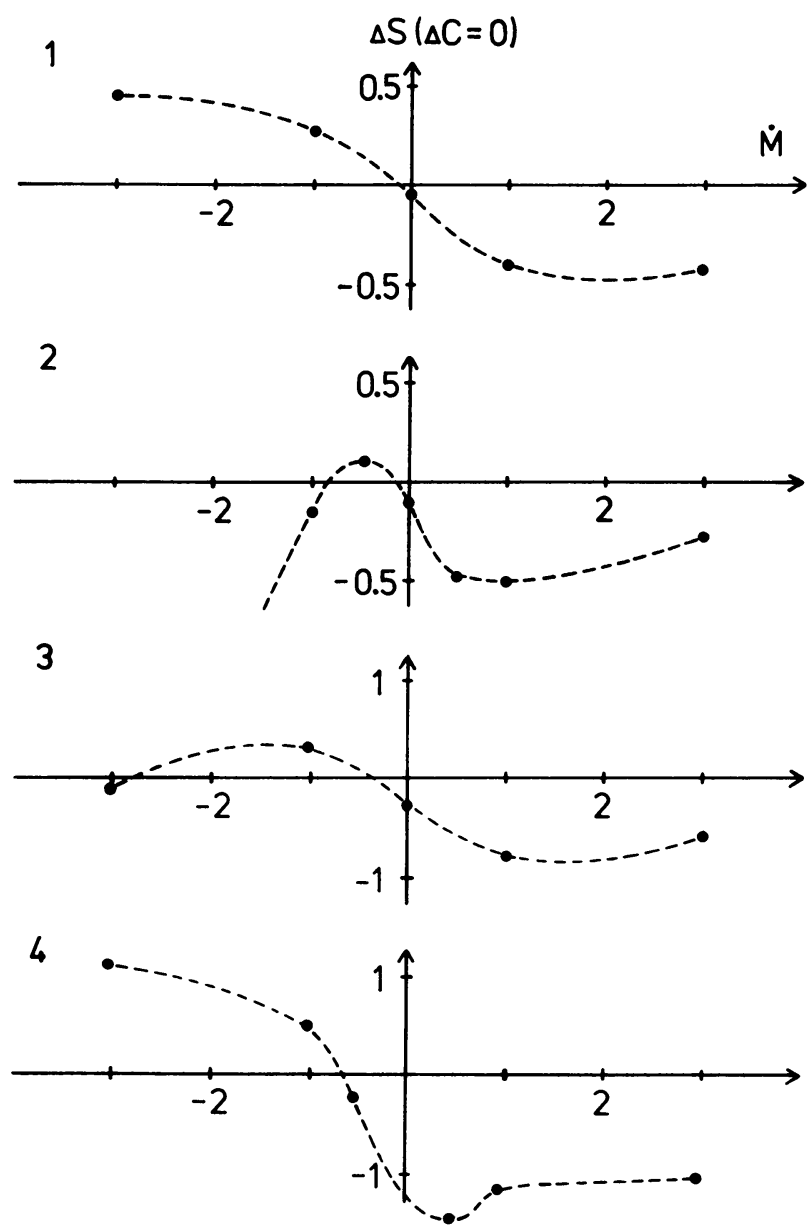

Fig. 1. Optimal values of $\Delta S$ (giving $\Delta C=0)$ for different rates of mass exchange $\dot{M}\left(10^{-\rightarrow} M_{\odot} \mathrm{yr}^{-1}\right)$ calculated by similar methods to those of Hazlehurst and Meyer-Hofmeister (1973). $\Delta S=\left(S_{\mathrm{p}}-S_{\mathrm{s}}\right)_{2}$ $-\left(S_{\mathrm{p}}-S_{\mathrm{s}}\right)_{1}$ and $\Delta C=\left(C_{\mathrm{p}}-C_{\mathrm{s}}\right)_{2}-\left(C_{\mathrm{p}}-C_{\mathrm{s}}\right)_{1}$ where 1 and 2 correspond to the points where $0.02 M_{\odot}$ and $0.04 M_{\odot}$ is transferred, respectively.

$$
\begin{aligned}
& \text { System 1: } 1.2 M_{\odot}+1.0 M_{\odot}, X_{\mathrm{cp}}=0.45 \\
& \text { System 2: } 1.2 M_{\odot}+0.6 M_{\odot}, X_{\mathrm{cp}}=0.40 \\
& \text { System 3: } 1.35 M_{\odot}+0.8 M_{\odot}, X_{\mathrm{cp}}=0.35 \\
& \text { System 4: } 1.5 M_{\odot}+1.1 M_{\odot}, X_{\mathrm{cp}}=0.59 \\
& X=0.7, Z=0.03, \alpha=1.5 .
\end{aligned}
$$


presumes a correctly chosen time step). The starting point (no gravitational terms) may also influence the results. For these reasons it was necessary to evolve the components simultaneously and for a longer time, in order that some definite conclusions could be drawn.

In the second step of the present work the system 1 of Figure $1\left(1.2 M_{\odot}+1.0 M_{\odot}\right)$ was chosen. Both components were evolved simultaneously and the solutions were coupled by iteration to give $S_{\mathrm{p}}=S_{\mathrm{s}}$ and $C_{\mathrm{p}}=C_{\mathrm{s}}$ at each time. Gravitational energy terms were included in both components. The parameter $C$, characterizing the equipotential surface, was calculated in a similar way to Moss (1971). About 10 evolutionary tracks were computed using different time steps. Independently of the time step used, excluding some numerical scatter, one of the three evolutionary tracks always resulted. The results are presented in Table I and Figure 2.

Track 1 clearly corresponds to the solutions of Moss and Track 2 to that found by $\mathrm{H} \& \mathrm{M}-\mathrm{H}$. Always the mass exchange accelerated during the evolution, but to a lesser extent for the Track 1 where the values of $\dot{M}$ are smallest (and hence also the gravitational effects smallest). In all cases the calculations were stopped when difficulty in finding new models appeared. So far it is not clear how sensitive the solutions are to the starting model. However, it seems that at least three different solutions exist for the case studied and under the assumptions made.

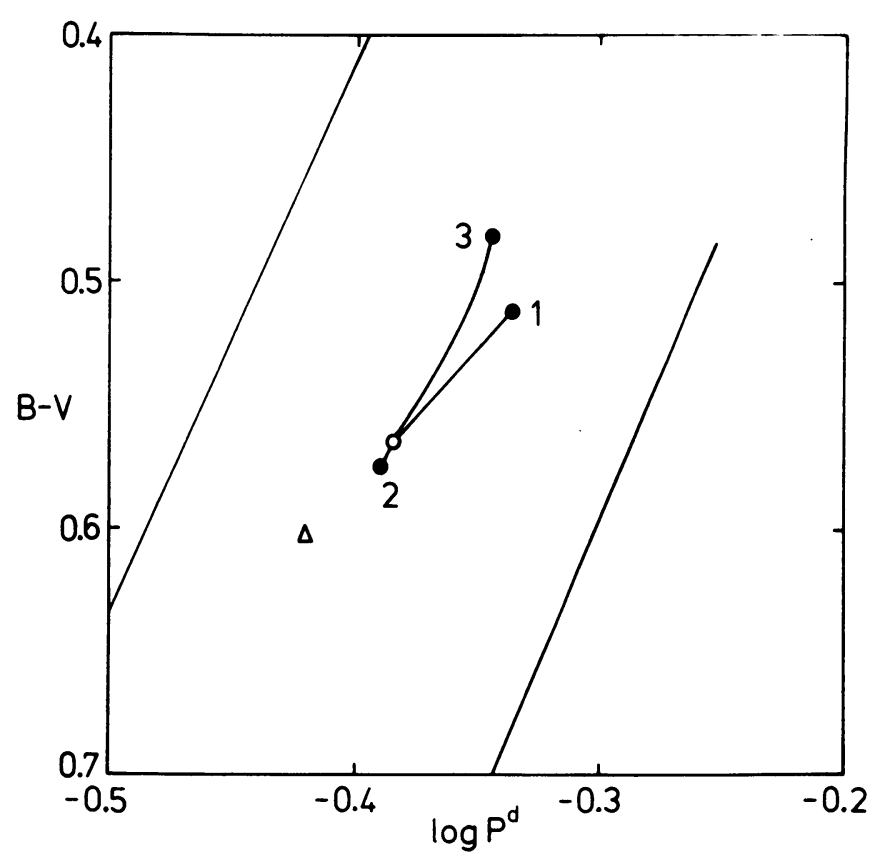

Fig. 2. Three evolutionary tracks of the initial system $1.2 M_{\odot}+1.0 M_{\odot}$ (open circle) in the periodcolour diagram (see Table I). The end points are marked by filled circles. Both components were evolved simultaneously to keep at each time $S_{\mathrm{p}}=S_{\mathrm{s}}$ and $C_{\mathrm{p}}=C_{\mathrm{s}}$. Time scales: Track $14.5 \times 10^{\mathrm{g}} \mathrm{yr}$,

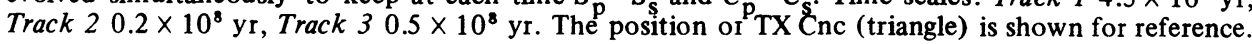




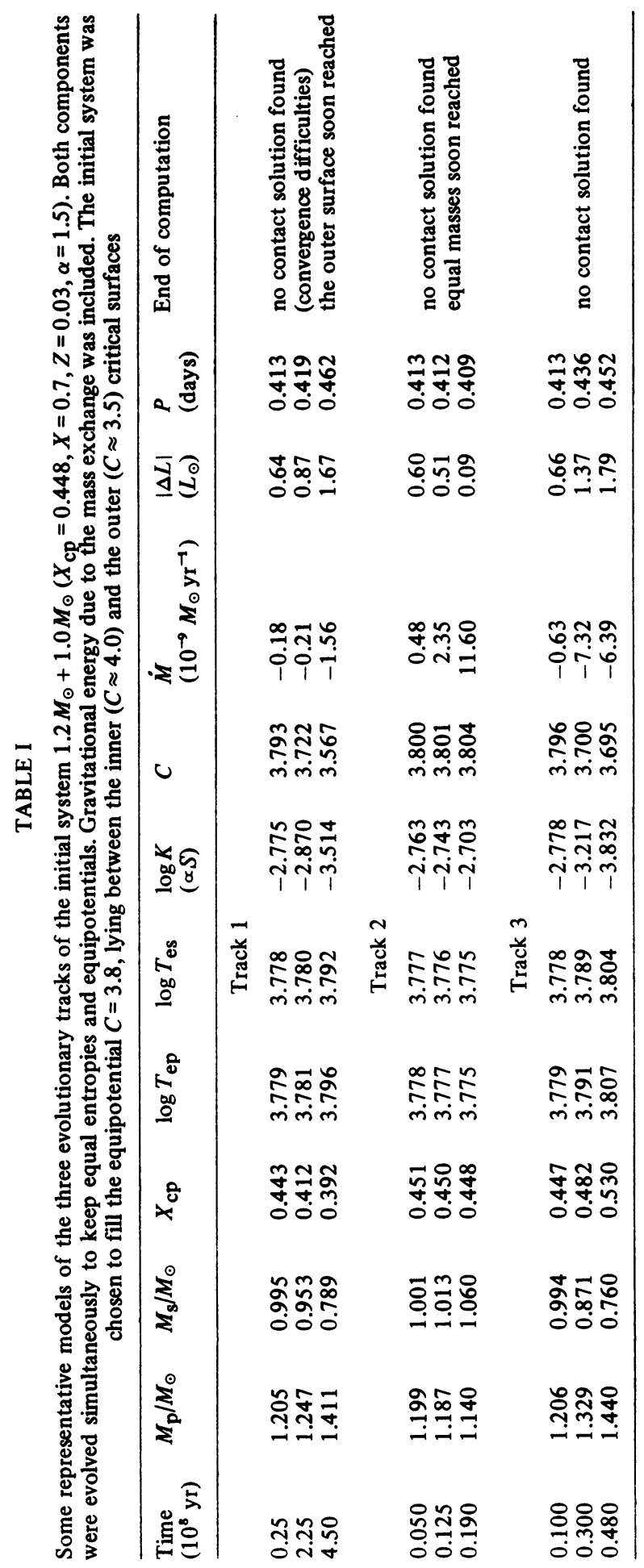


If one wants to apply the models calculated for the numerous W UMA-systems, the Track 1 type of evolution might seem most promising due to its longest time scale. However, to get any definite answer in this respect, a stability analysis of the models is needed to find out which track the evolution really chooses. Further, it was assumed that the surface of the common envelope can freely move between the outer and inner critical surfaces. If only one fixed equipotential surface would be allowed (say the inner critical Roche lobe), then the Track 2 seems to be the only possibility because for it the equipotential surface remained remarkably constant. On the other hand, the exact position of the starting surface (the initial value of $C$ ) seems to be unimportant. However, it should not be too close to the outer critical surface.

How realistic our starting models are depends on how rapidly the initial conditions, especially the equal entropies, are reached after the first contact. For this much more knowledge about the luminosity transfer mechanism and about the origin of such systems is needed.

We can also speculate that the actual track oscillates randomly (c.f. period changes in W-systems) between the solutions. It seems probable that the three possibilities always exist, independently of the actual model at that moment. Similar solutions may exist also for zero age systems. We should only take sufficiently large mass transfer rates $(\dot{M})$ to fulfil the contact conditions, which otherwise would be impossible.

\section{References}

Hazlehurst, J. and Meyer-Hofmeister, E.: 1973, Astron. Astrophys. 24, 379.

Moss, D. L.: 1971, Monthly Notices Roy. Astron. Soc. 153, 41.

\section{DISCUSSION}

Hazlehurst: I have the following comments. (1) It seems that we have here a well-posed initial value problem, so I don't see how there can be more than one possible solution. (2) If you take out the 'gravitational' terms you make it impossible for the star to evolve on a rapid time scale; if you leave them in, the star still has the choice to evolve slowly if it wants to. (3) If we are really in a thermally unstable situation, the matter will go whichever way it is pushed - in this case it is the primary which pushes, since it is the more rapidly evolving star.

Whelan: The direction of mass exchange in contact binary evolution is not obvious just from thinking about it. Plausibly we can say the primary evolves and wants to increase radius and luminosity which would tend to drive mass from primary to secondary. But the luminosity increase may increase $\Delta L$ (the transferred luminosity) which may increase the secondary; radius and temperature causing a tendency for mass flow from secondary to primary. Which wins must be found from detailed computation.

Webbink: Can you identify, in any physical sense, the reasons why three divergent evolutionary possibilities should exist?

Vilhu: I think that the situation is quite natural. We have here an additional parameter $\dot{m}$ which carries gravitational energy and influences in opposite ways the mass losing and mass accreting components, if it is sufficiently large. Further, allowing the equipotential surface to move between the inner and outer surfaces, we can get solutions for large negative and positive $\dot{m}$ (Note that the potential surface naturally does not behave similarly in these cases). The slowest solution (small negative $\dot{m}$, and hence also negligible gravitational effects) can also be shown to exist using only thermal equilibrium models.

Moss: My models did include gravitational terms for primary but they were effectively reduced to zero by looking for evolution on long timescale, and taking long timesteps. 